\title{
Mechanically coupled clamped circular plate resonators: modeling, design and experimental verification
}

\author{
Muhammed Abdulcelil Acar ${ }^{1, *} \mathbb{B}$, Abdullah Atalar ${ }^{1,2}$, Mehmet Yilmaz ${ }^{2,3}$ \\ and Hayrettin Köymen ${ }^{1,2}$ \\ ${ }^{1}$ Department of Electrical and Electronics Engineering, Bilkent University, 06800 Ankara, Turkey \\ 2 UNAM-National Nanotechnology Research Center, Bilkent University, 06800 Ankara, Turkey \\ ${ }^{3}$ Institute of Materials Science and Nanotechnology, Bilkent University, 06800 Ankara, Turkey \\ E-mail: acar@ee.bilkent.edu.tr
}

Received 2 May 2021, revised 30 June 2021

Accepted for publication 11 August 2021

Published 27 August 2021

\begin{abstract}
Mechanically coupled resonators usually require a mechanical coupling element; this introduces additional complexity to the picture. We propose a novel modeling and design approach, followed by experimental verification, for mechanically coupled clamped circular plate resonators in which no additional coupling element is present. In our study, the flexural mode clamped circular plate resonators overlap to an extent, and their clamps at the overlap region are removed to generate a freely moving coupling boundary between the resonators. The practical measure of the overlapping is small enough to preserve the characteristics of each resonator. This result enables modeling the coupled resonators based on the clamped circular plate resonator model. A physics-based lumped element equivalent circuit model is developed where dimensions, bias voltage, and material properties are controllable variables. Each of the model parameters is expressed as the corresponding single resonator model parameter multiplied by a function of the amount of overlap. Analytical derivations and finite element method simulations are used to extract the dependency of the model parameters on the amount of overlap. Closed-form expressions for center frequency, bandwidth, and termination impedance of the coupled resonator are derived using the developed model. A design procedure is introduced to determine dimensional parameters and bias voltage. The proposed coupled-resonator offers up to $5 \%$ fractional bandwidth. For a typical design using a polysilicon plate with $100 \mathrm{~nm}$ gap height, the ratio of the termination impedance to the center frequency is calculated to be $158 \Omega \mathrm{MHz}^{-1}$. This result indicates that on-chip intermediate frequency filters can be implemented at center frequencies up to several $100 \mathrm{MHz}$ using this type of coupled resonators. A coupled resonator is designed and realized for a proof of concept demonstration. The measurement results of the coupled resonator show good agreement with the equivalent circuit model simulations.
\end{abstract}

Keywords: circuit modeling, clamped circular plate resonator, coupled resonators, on-chip IF filter

(Some figures may appear in colour only in the online journal)

\footnotetext{
* Author to whom any correspondence should be addressed.
} 


\section{Introduction}

Mechanical filters date back to the late 1930s [1]. Taking advantage of the small form factor and high-Q of mechanical resonators, these types of filters became the replacement of the lumped element electronic filters. The operation principle of a mechanical filter is as follows: electrical input signals are converted to mechanical energy by a transducer. Mechanical resonators coupled via mechanical rods perform filtering in the mechanical domain. A second transducer is used to transform the filtered mechanical signals into electrical output. Magnetostrictive materials were employed to be the transducers of early designs [1-3]. Piezoelectric transducers emerged later [4-6] due to their superior conversion efficiency and reliability. Analytical derivations, circuit design methods, and application examples for common mechanical filter types were given in [7]. A new era started for mechanical filters with the emergence of commercially available MEMS technology more than two decades ago. Small-scale, high-Q, micro-electromechanical resonators based on CMOS compatible processes enabled on-chip integration of MEMS filter structures with electronic circuitry [8-11].

Lin et al [12], Wang and Nguyen [13], and Jing et al [14] introduced mechanically coupled comb-transduced resonator filters. These resonators move laterally and beam springs provide mechanical coupling. This type of filter is vulnerable to fabrication tolerances due to its mechanical complexity. Also, the highest center frequency achievable is in the order of $1 \mathrm{MHz}$. On the other hand, Bannon et al [15] and Motiee et al [16] used beam coupled clamped-clamped beam resonators. Compared to the mechanically coupled comb-transduced resonators, these coupled resonators have relatively lower mechanical complexity and offer up to ten times higher center frequencies.

Pourkamali and Ayazi [17, 18] and Galayko et al [19] introduced non-mechanical coupling, either capacitive or electrostatic, between clamped-clamped beam resonators. There are two main advantages of the non-mechanical coupling approach. The first advantage is lower mechanical complexity. The second advantage is the post-production tuning capability of the coupling strength. However, it is important to emphasize that the second advantage requires the necessity to control the independent bias voltages of the resonators.

Li et al [20, 21] and Shalaby et al [22] reported bulk acoustic mode resonator-based filters. The bulk acoustic mode resonators exhibit higher effective stiffness than their flexural mode counterparts, which makes them suitable for higher frequency applications. Mechanically coupled radial-contour mode disk arrays were demonstrated for reducing the termination impedance and suppression of unwanted spurious modes [20, 21].

In [12-22], the conceptual lumped element electrical circuit analogies are used to predict the performance of the resonators and filters. Equivalent circuit model parameters are arbitrarily tuned according to the measurement results. By employing these adjusted element values, the comparable performance of equivalent circuits is demonstrated. This kind of modeling approach is not useful for designing new structures since the model elements are not represented by the correct functions of the design variables.

Greywall and Busch [23] presented mechanically coupled drumhead resonator filters. The input resonator is electrically driven using a circular ring electrode, and the displacement amplitude of the output resonator is sensed making use of optical interference techniques. The cyclic connections of up to 20 coupled resonators were demonstrated, and a symmetric bandpass filter response was achieved without the need for post-production tuning.

In this paper, we investigate the properties of mechanically coupled clamped circular plate resonators. We provide analytical derivations, which determine the practical limits of these coupled resonators for on-chip filter applications.

We use a modified version of the small-signal equivalent circuit model of the capacitive micromachined ultrasonic transducer given in [24] that was developed for a single clamped circular plate resonator. The developed single resonator model is very accurate and comparable to finite element method (FEM) for any given set of dimensions, bias voltage, and material properties.

We develop a coupled-resonator model based on the single resonator model and the capacitive coupling approach. Model parameters of the coupled resonators are expressed in terms of the single resonator model parameters and the amount of overlap between resonators using analytical derivations and FEM simulation results. Since we do not repeat the FEM simulations used for the model development, the model implemented in a circuit simulator allows for fast optimization of design parameters for given specifications, like center frequency, bandwidth, and optimal termination impedance.

In our coupled-resonator approach, the coupling mechanism does not require any additional mechanical element similar to the structure used in [23] and unlike the commonly used coupled MEMS resonator structures. Instead, the clamped circular plate resonators are deliberately overlapped to form the mechanical coupling generated at the interface. Compared to the coupled resonators with a separate mechanical coupling element, the mechanical complexity is reduced, and the reliability is increased. The resulting structure differs from the one given in [23] due to the shape of the electrodes and the type of output. We prefer to use full circular electrodes, rather than the ring-shaped electrodes used in [23], to achieve higher excitation efficiency of the fundamental mode resonance and a higher electromechanical transformation ratio, which reduces the termination impedance value. In order to allow proper integration with electronic circuitry, an electrical output is implemented instead of the optical interference techniquebased output detection method described in [23].

The spatial velocity of the vibrating clamped circular resonator plate decreases as the radial location becomes distant from the central point and goes to zero at the clamped periphery. The proposed structure inherently provides low-velocity coupling [25] between the resonators without the need for a mechanical coupling element. The low-velocity coupling methodology allows us to achieve narrower filter bandwidths compared to coupling the resonators at their maximum velocity points. 


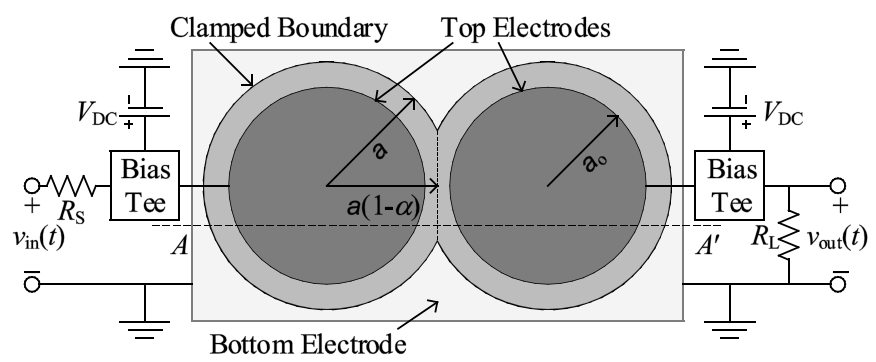

(a)

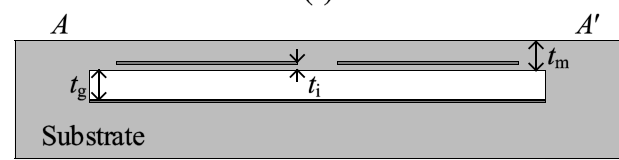

(b)

Figure 1. (a) Top view with geometrical details and $\mathrm{I} / \mathrm{O}$ electronic circuitry and (b) unbiased $A-A^{\prime}$ cross-sectional view of the proposed mechanically coupled clamped circular plate resonators.

We implement a coupled-resonator design based on a commercial process. Although the gap height and resonator thickness options provided in this process limit the lowest termination impedance, this process is used to demonstrate the validity of our model. Measurement results are in good agreement with our model predictions.

\section{Coupled-resonator structure}

We propose a new coupled micromechanical resonator structure. The clamped circular plate is selected as the resonator unit. Two resonator plates are overlapped, i.e. overlap ratio $(\alpha)$ defined in figure 1 is between 0 and 0.1 , and the clamps of each resonator at the overlapped region are removed in order to form the coupling mechanism. In other words, the resonator plates are mechanically connected and free to move at the overlapping region. Analytical expression of a practical parameter called overlap area ratio $(\Delta)$, i.e. the overlap area divided by the original circular resonator area, is

$$
\Delta=\frac{1}{\pi}\left(\cos ^{-1}(1-\alpha)-(1-\alpha) \sin \left(\cos ^{-1}(1-\alpha)\right)\right) .
$$

When an electrical signal is applied between the input resonator plate and the bottom electrode, the resulting electrostatic forces vibrate the input resonator plate. The generated mechanical energy is transferred to the output resonator plate through the mechanical coupling interface and converted back to an electrical signal between the output resonator plate and the bottom electrode. The overall electrical signal transfer process is frequency selective since the coupled-resonator structure has two fundamental resonances. Cross-sectional views of these resonance mode shapes are illustrated in figure 2 . The lower frequency resonance corresponds to the out-of-phase movement of the resonators. On the other hand, the higher frequency resonance happens when the resonators are moving in phase. Normalized peak displacements and phases of the input

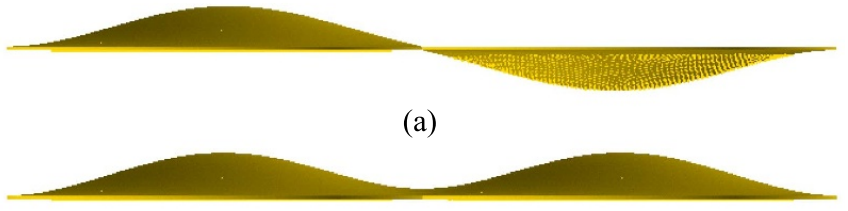

(b)

Figure 2. Cross-sectional views of (a) out-of-phase and (b) in-phase mode shapes of the proposed mechanically coupled clamped circular plate resonators.

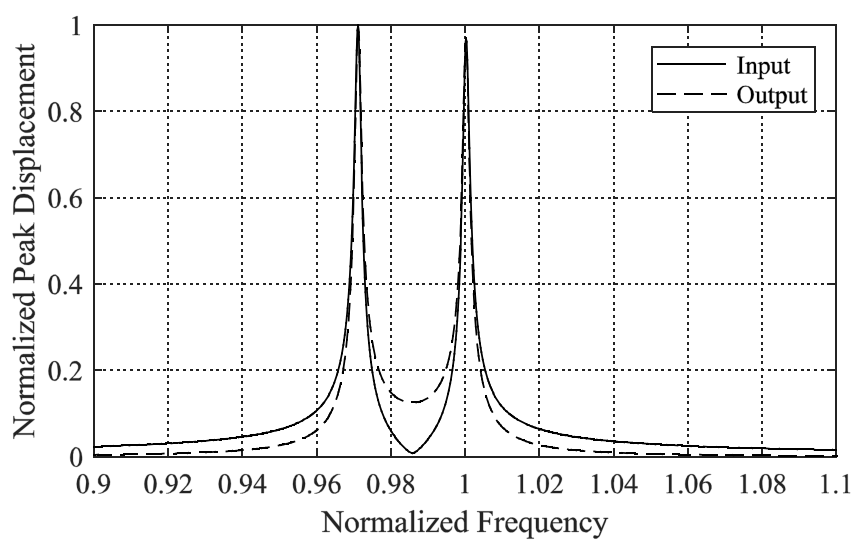

(a)

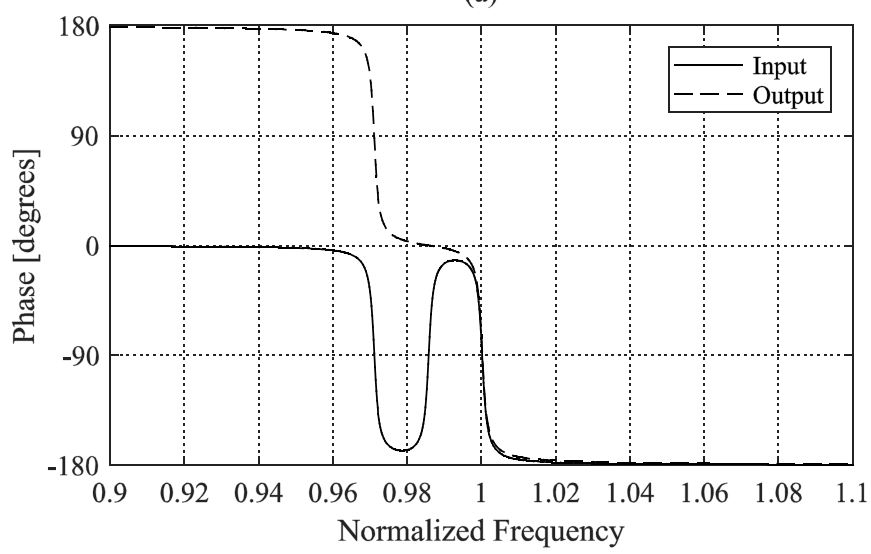

(b)

Figure 3. (a) The normalized peak displacements and (b) the phases of input and output resonators as a function of normalized frequency, for $\alpha=0.05$.

and output resonators as functions of frequency are plotted in figure 3. The frequency axis is normalized with the fundamental resonance frequency of a single and fully clamped circular plate resonator, which is a reference resonator.

\section{Small-signal equivalent circuit model}

We introduce a small-signal equivalent circuit model for the resonators, which is valid for the overlap ratio range $0<\alpha<0.1$. Altering the overlap ratio adjusts the coupling coefficient between the two resonators and determines the fractional bandwidth up to $5 \%$. We define each model 


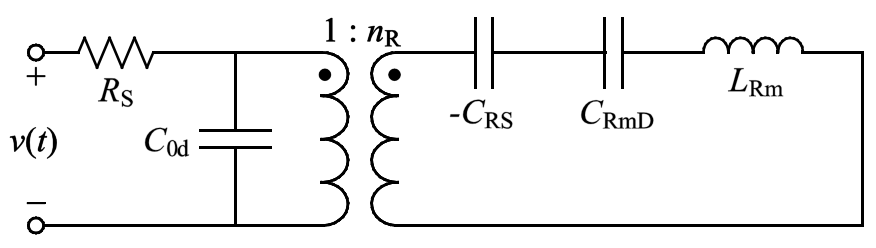

Figure 4. Small-signal equivalent circuit model of a single clamped circular plate resonator under vacuum.

parameter as a function of the corresponding single resonator model parameter and the overlap ratio.

\subsection{Single resonator model}

The small-signal equivalent circuit model of a single clamped circular plate resonator is shown in figure 4 [24]. We introduce the RMS dynamic compliance $\left(C_{\mathrm{RmD}}\right)$ parameter into the existing single resonator model. $C_{\mathrm{RmD}}$ is approximately $2.2 \%$ higher than $C_{\mathrm{Rm}}$ which is the RMS static compliance of the clamped circular plate. $C_{\mathrm{Rm}}$ is calculated under uniform static force distribution assumption which results in a static displacement profile [26-28] given as

$$
X(r)=X_{\mathrm{P}}\left(1-\left(\frac{r}{a}\right)^{2}\right)^{2} \quad \text { for } r \leqslant a,
$$

where $X_{\mathrm{P}}$ is the peak static displacement under $V_{\mathrm{DC}}$ bias voltage. On the other hand, $C_{\mathrm{RmD}}$ is determined by the fundamental resonance frequency for a clamped circular plate obtained using plate theory $[29,30]$, as

$$
f_{0}=\frac{1}{2 \pi \sqrt{L_{\mathrm{Rm}} C_{\mathrm{RmD}}}}=0.469 \frac{t_{\mathrm{m}}}{a^{2}} \sqrt{\frac{Y_{0}}{\left(1-\sigma^{2}\right) \rho}},
$$

where $Y_{0}$ is Young's modulus, $\sigma$ is Poisson's ratio, and $\rho$ is the mass density of the plate.

\subsection{Coupled-resonator model}

The equivalent circuit model of the coupled resonator is shown in figure 5. The mechanical coupling between the resonators can be represented with a shunt capacitance $C_{\mathrm{R} 2}$ between the equivalent circuit models of the resonators. $C_{\mathrm{R} 1}$ is the compliance of each resonator.

The mass of each resonator plate is modified due to the resonator area reduction as

$$
L_{\mathrm{Rm}}^{\prime}=L_{\mathrm{Rm}}(1-\Delta) .
$$

Based on the FEM simulations and relative to the single resonator model parameters, extracted small-signal equivalent circuit model parameters of the electrical input capacitance, RMS electromechanical turns ratio, and RMS spring softening capacitance turn out to be

$$
\begin{gathered}
C_{0 \mathrm{~d}}^{\prime} \approx C_{0 \mathrm{~d}}, \\
n_{\mathrm{R}}^{\prime} \approx n_{\mathrm{R}} \sqrt{(1-\Delta)},
\end{gathered}
$$

and

$$
C_{\mathrm{RS}}^{\prime} \approx \frac{C_{\mathrm{RS}}}{(1-\Delta)}
$$

having less than $0.5 \%$ error for the overlap ratio range $0<\alpha<0.1$ and $V_{\mathrm{DC}} / V_{\mathrm{r}} \leqslant 0.9$

$$
V_{\mathrm{r}}=8 \frac{t_{\mathrm{m}}^{3 / 2}}{a^{2}} t_{\mathrm{ge}}^{3 / 2} \sqrt{\frac{Y_{0}}{27 \varepsilon_{0}\left(1-\sigma^{2}\right)}},
$$

is the collapse voltage of a single resonator with full electrodes under vacuum, where $t_{\mathrm{ge}}=t_{\mathrm{g}}+t_{\mathrm{i}} / \varepsilon_{\mathrm{r}}, \varepsilon_{0}$ is the permittivity of free space and $\varepsilon_{\mathrm{r}}$ is the relative permittivity of the insulating material.

The mechanical compliance parameters $C_{\mathrm{R} 1}$ and $C_{\mathrm{R} 2}$ can be approximated by third order polynomial fit to the FEM simulation results for the overlap ratio range $0<\alpha<0.1$ as

$$
C_{\mathrm{R} 1} \approx \frac{C_{\mathrm{RmD}}(1-\Delta)}{1-1.20 \alpha-0.305 \alpha^{2}+14.5 \alpha^{3}}
$$

and

$$
C_{\mathrm{R} 2} \approx \frac{C_{\mathrm{RmD}}(1-\Delta)}{\alpha\left(0.596-2.03 \alpha-13.6 \alpha^{2}\right)},
$$

where the $C_{\mathrm{RmD}}$ term covers the dependence on the material properties and the resonator plate parameters. As the overlap ratio $(\alpha)$ goes to 0 , the coupling between the resonators becomes negligible and the coupled-resonator model in figure 5 approaches the model of two uncoupled resonators.

The small-signal equivalent circuit model given in figure 5 is implemented in a circuit simulator and verified for several different dimensional parameters by comparing the circuit simulator results with the FEM simulation results.

\section{Coupled-resonator design}

\subsection{Defining design specifications}

The out-of-phase and the in-phase resonance frequencies of the coupled-resonator structure can be expressed as:

$$
f_{1}=\frac{1}{2 \pi} \sqrt{\frac{1}{L_{\mathrm{Rm}}^{\prime}}\left(\frac{1}{C_{\mathrm{R} 1}}-\frac{1}{C_{\mathrm{RS}}^{\prime}}\right)}
$$

and

$$
f_{2}=\frac{1}{2 \pi} \sqrt{\frac{1}{L_{\mathrm{Rm}}^{\prime}}\left(\frac{1}{C_{\mathrm{R} 1}}+\frac{2}{C_{\mathrm{R} 2}}-\frac{1}{C^{\prime}{ }_{\mathrm{RS}}}\right)} .
$$

We define the center frequency as $f_{\mathrm{c}}=0.5\left(f_{1}+f_{2}\right)$, which shifts down as the overlap ratio and DC bias voltage increase. The bandwidth is assumed to be the frequency difference between in phase and out of phase resonance modes, i.e. $B=f_{2}-f_{1}$. Unlike the center frequency, the bandwidth increases as the DC bias increases. The bias voltage of the resonators is set to $V_{\mathrm{DC}}=0.9 V_{\mathrm{r}}$, considering the trade-off 


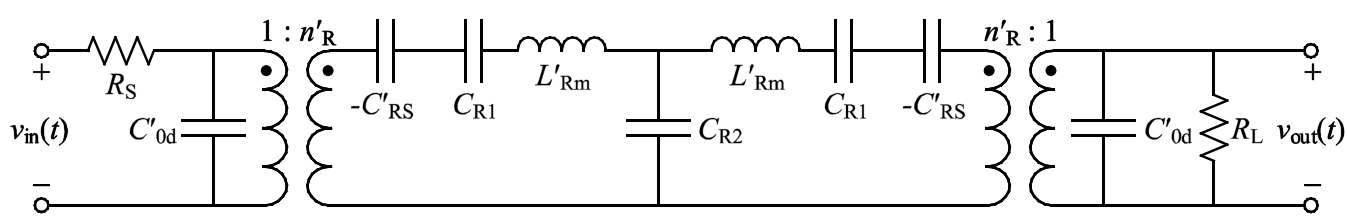

Figure 5. Small-signal equivalent circuit model of the coupled resonator structure.

between termination impedance and linearity. We can approximate the center frequency and the bandwidth for the overlap ratio range $0<\alpha<0.1$ under $a_{0}=0.8 a$ and $V_{\mathrm{DC}} / V_{\mathrm{r}}=0.9$ conditions as

$$
f_{\mathrm{c}} \approx f_{0}\left(0.784-0.333 \alpha+0.812 \alpha^{2}-6.39 \alpha^{3}\right)
$$

and

$$
B \approx f_{\mathrm{c}} \alpha\left(0.953-1.70 \alpha-31.3 \alpha^{2}\right) .
$$

The fractional bandwidth, $B / f_{\mathrm{c}}$, reaches its maximum value of $5 \%$ at $\alpha \approx 0.084$.

Mechanical resonator structures suffer from their high electrical input impedance characteristics. Several studies were reported on the reduction of the input impedance $[15,20,21,31-38]$. We derived a closed form analytical expression of the electrical termination impedance $\left(Z_{0}\right)$ in order to understand how it is related to the design parameters. Referring to figure 5 , the termination impedance, $Z_{0}$, is obtained under the $R_{\mathrm{S}}=R_{\mathrm{L}}=Z_{0}$ condition as

$$
Z_{0}=\frac{2 \pi L_{\mathrm{Rm}}^{\prime}}{n^{\prime}{ }_{\mathrm{R}}{ }^{2}} \frac{\sqrt{\left(f_{\mathrm{c}}^{2}-f_{1}^{2}\right)\left(f_{2}^{2}-f_{\mathrm{c}}^{2}\right)}}{f_{\mathrm{c}}} .
$$

We can approximate $Z_{0}$ using (5), (7), (15), and narrowband filter assumption, i.e. $B / f_{\mathrm{c}} \ll 1$, as

$$
Z_{0} \approx \frac{2 \pi L_{\mathrm{Rm}} B}{n_{\mathrm{R}}^{2}}
$$

For an electrode radius $a_{0}=0.8 a$ and $V_{\mathrm{DC}} / V_{\mathrm{r}}=0.9$, we can rearrange (16) as either

$$
Z_{0} \approx \frac{1.08}{\varepsilon_{0}} \frac{\rho\left(1-\sigma^{2}\right)}{Y_{0}}\left(\frac{a}{t_{\mathrm{m}}}\right)^{2} t_{\mathrm{ge}} B
$$

or

$$
Z_{0} \approx \frac{1}{2 \varepsilon_{0}} \sqrt{\frac{\rho\left(1-\sigma^{2}\right)}{Y_{0}}} \frac{t_{\mathrm{ge}}}{t_{\mathrm{m}}} \frac{B}{f_{0}}
$$

For the specific case of $t_{\mathrm{ge}}=100 \mathrm{~nm}, a / t_{\mathrm{m}}=10, \alpha=0.01$, and the polysilicon resonator plate, the ratio of the termination impedance to the center frequency, $Z_{0} / f_{\mathrm{c}}$, becomes $158 \Omega \mathrm{MHz}^{-1}$.

\subsection{Design procedure}

To design a second-order bandpass filter with center frequency, $f_{\mathrm{c}}$, and bandwidth, $B$, using termination impedances of $Z_{0}$, the design procedure is as follows:

(1) Set $a_{0}=0.8 a$ to avoid the short circuit between the electrodes for the overlap ratio range $0<\alpha<0.1$.

(2) Set $V_{\mathrm{DC}} / V_{\mathrm{r}}=0.9$ to achieve the lowest practical termination impedance.

(3) Set $a / t_{\mathrm{m}}=10$ to achieve as low a termination impedance as possible considering the model validity.

(4) Find the overlap ratio $\alpha$ using (14).

(5) Determine $a$ and $t_{\mathrm{m}}$ using $a / t_{\mathrm{m}}=10,(3),(13)$, and material properties of the resonator plate.

(6) Calculate $t_{\mathrm{ge}}$ using (17), $B$, and material properties of the resonator plate.

(7) Find $V_{\mathrm{DC}}$ using (8) and $V_{\mathrm{DC}} / V_{\mathrm{r}}=0.9$.

(8) Fine-tune the parameters to achieve the desired response using the circuit simulator, making sure that $a / t_{\mathrm{m}} \geqslant 10$.

For example, a bandpass filter made on polysilicon material with $f_{\mathrm{c}}=127 \mathrm{MHz}, B=5 \mathrm{MHz}$, and $Z_{0}=17 \mathrm{k} \Omega$ has $t_{\mathrm{ge}}=20 \mathrm{~nm}, a=2.4 \mu \mathrm{m}, t_{\mathrm{m}}=240 \mathrm{~nm}, \alpha=0.05$, and $V_{\mathrm{DC}}=11 \mathrm{~V}$. Another design on the same material with $f_{\mathrm{c}}=107 \mathrm{MHz}, B=1 \mathrm{MHz}$, and $Z_{0}=3.4 \mathrm{k} \Omega$ has $t_{\mathrm{ge}}=20 \mathrm{~nm}$, $a=2.9 \mu \mathrm{m}, t_{\mathrm{m}}=290 \mathrm{~nm}, \alpha=0.01$, and $V_{\mathrm{DC}}=10 \mathrm{~V}$.

\section{An example design}

We used the PolyMUMPs process of MEMSCAP ${ }^{4}$ and designed a coupled-resonator unit for a proof of concept demonstration. PolyMUMPs is a three-layer polysilicon surface micromachining and bulk micromachining process, with two sacrificial layers and one metal layer. This process is based on a silicon substrate and has a minimum feature size of $2 \mu \mathrm{m}$. A $600 \mathrm{~nm}$ thick silicon nitride layer is deposited on the substrate to provide electrical isolation between the polysilicon layers and the substrate. The first polysilicon layer, Poly0, is $500 \mathrm{~nm}$ thick and takes place on top of the nitride layer. Then, a $2 \mu \mathrm{m}$ phosphosilicate glass (PSG) sacrificial layer (Oxide1) deposition follows the photolithography patterning of the Poly0 layer. The structural layers of polysilicon (Poly1 and Poly2) are $2 \mu \mathrm{m}$ and $1.5 \mu \mathrm{m}$ thick, respectively. Table 1 lists the material properties of the structural layers. In between the two structural layers, a $750 \mathrm{~nm}$ PSG sacrificial layer (Oxide2) is deposited. (This sacrificial layer thickness

${ }^{4}$ MEMSCAP, S.A., Cedex, France, www.memscap.com. 
Table 1. Material properties of the polyMUMPs structural layers of polysilicon (Poly1 and Poly2).

\begin{tabular}{ll}
\hline Young's modulus, $Y_{0}$ & $158 \pm 10 \mathrm{GPa}$ \\
Density, $\rho$ & $2330 \mathrm{~kg} \mathrm{~m}^{-3}$ \\
Poisson ratio, $\sigma$ & $0.22 \pm 0.01$ \\
\hline
\end{tabular}
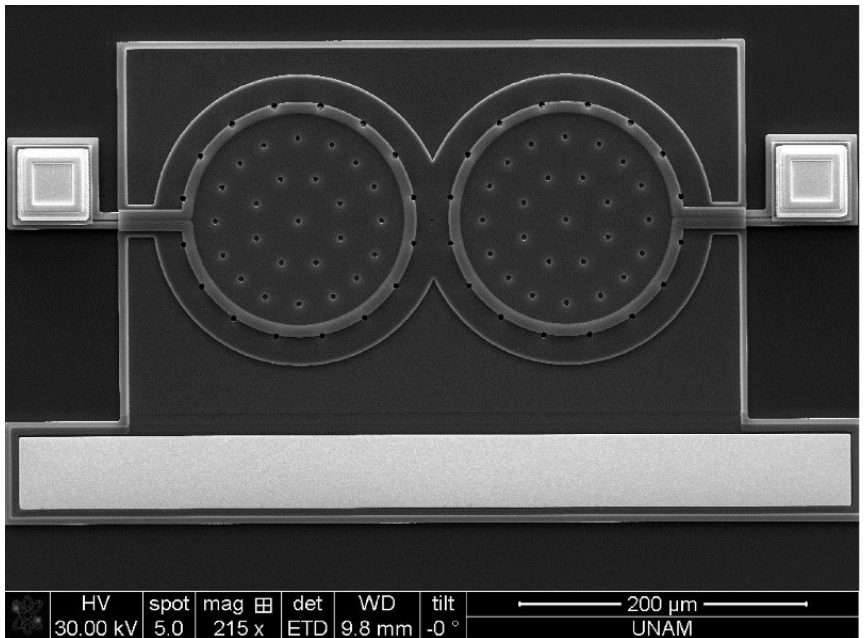

(a)

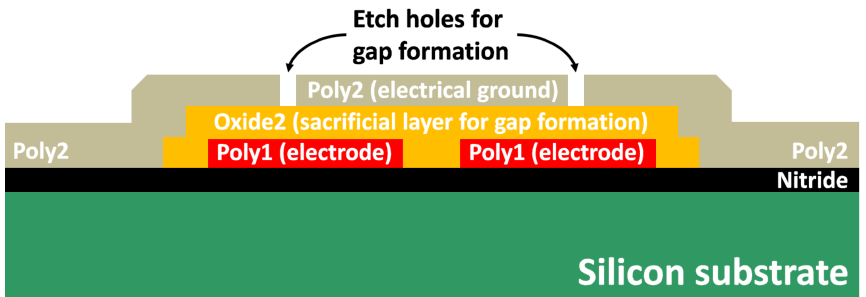

(b)

Figure 6. (a) Top view SEM image and (b) the cross-section illustration (not drawn to scale) of a coupled-resonator device based on the PolyMUMPs process.

forms the gap height, $t_{\mathrm{g}}$, of the resonator structure.) The final layer is $0.5 \mu \mathrm{m}$ gold, which provides an electrical interface for probing and bonding.

A top view SEM image and the cross-section illustration of a sample coupled-resonator structure are shown in figure 6 . We use the Oxide 2 layer for gap formation of the resonators since it provides the lowest possible gap height of $750 \mathrm{~nm}$. Since the PolyMUMPs process has conductive structural layers, we cannot have isolated electrodes on mechanically coupled resonator plates. Instead, as shown in figure 6(b), we form the isolated electrodes using the bottom layer (Poly1) on the substrate and connect the moving plate layer (Poly2) to the electrical ground. The resulting structure in figure 6(b) is functionally equivalent to the original proposal shown in figure 1. The coupled-resonator design does not include the Poly0 and Oxide1 layers and starts with the Poly1 layer, which is used for input and output electrodes on top of the nitride isolation layer. Then, the Oxide2 layer is placed where we require the gap. We have the Poly2 layer on top as the resonator plate material. As shown in figure 6(a), this Poly2 layer has holes with a $2 \mu \mathrm{m}$ radius and $30 \mu \mathrm{m}$ interval. These holes serve the removal of

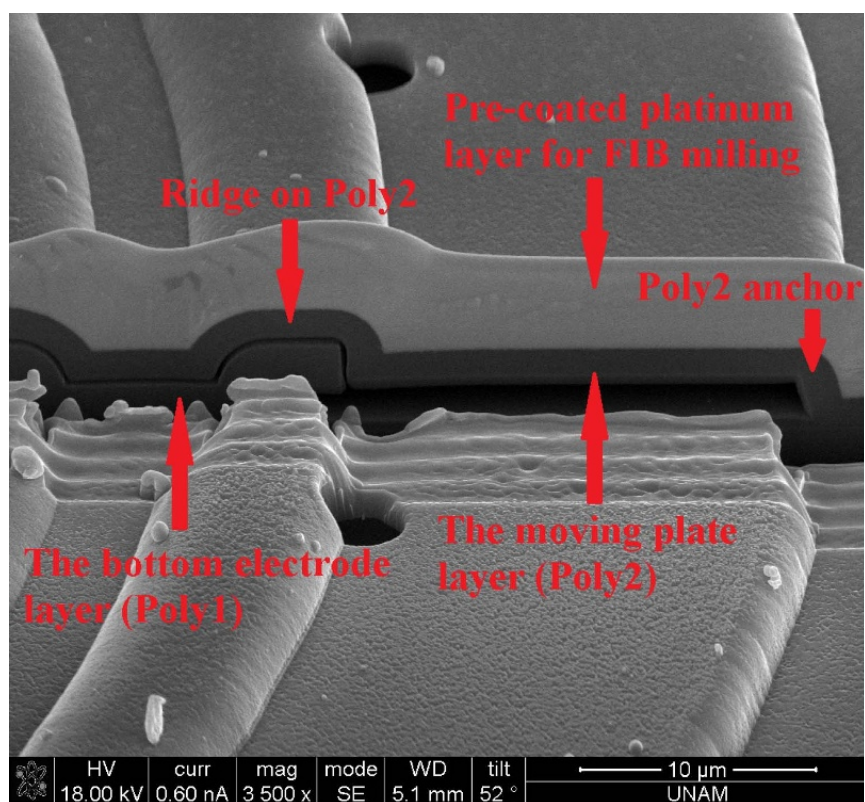

(a)

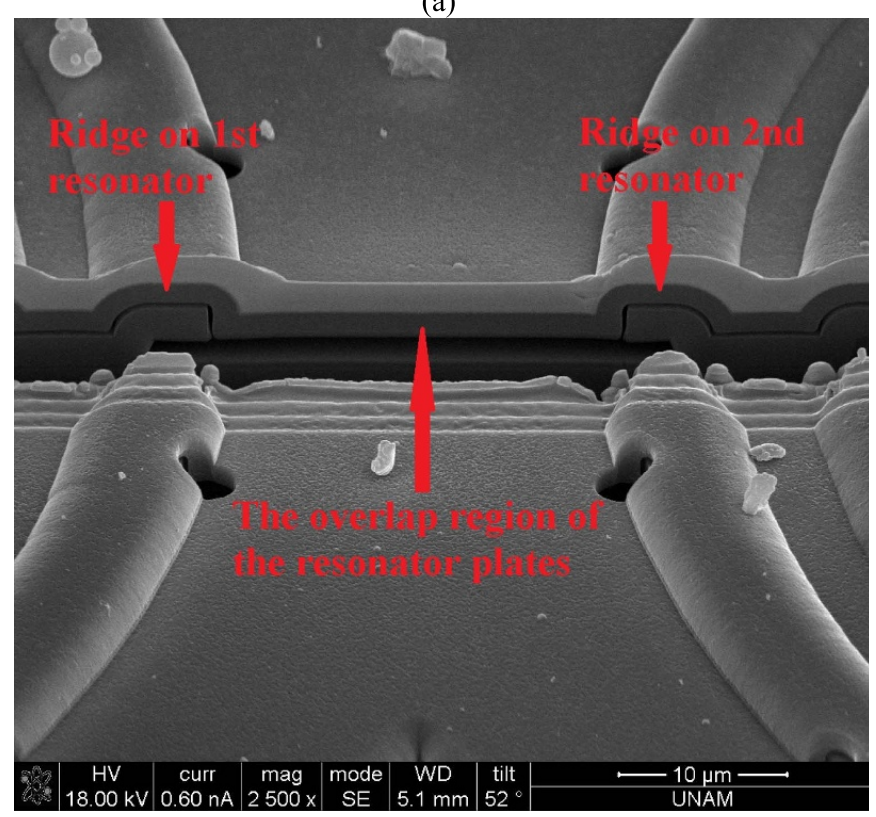

(b)

Figure 7. SEM views of FIB-cuts showing the cross-section of (a) the moving plate anchored to the substrate and (b) the overlap region including the moving plate and the bottom electrodes.

the sacrificial layer during the HF release process. The metal layer is used for wire bonding the input, output, and ground interconnection pads.

Although the coupled resonator unit explained in this section is valid for the proof of concept demonstration, some structural properties of the PolyMUMPs process are different from the model assumptions. We need to take these into account when comparing the model performance with the measurements. First, the Poly2 layer is not strictly clamped at the resonator periphery. Instead, as shown in figure 7(a), it is anchored to the substrate through the stack-up of Oxide 1 and Oxide2 layers. The second difference is due to the conformal 


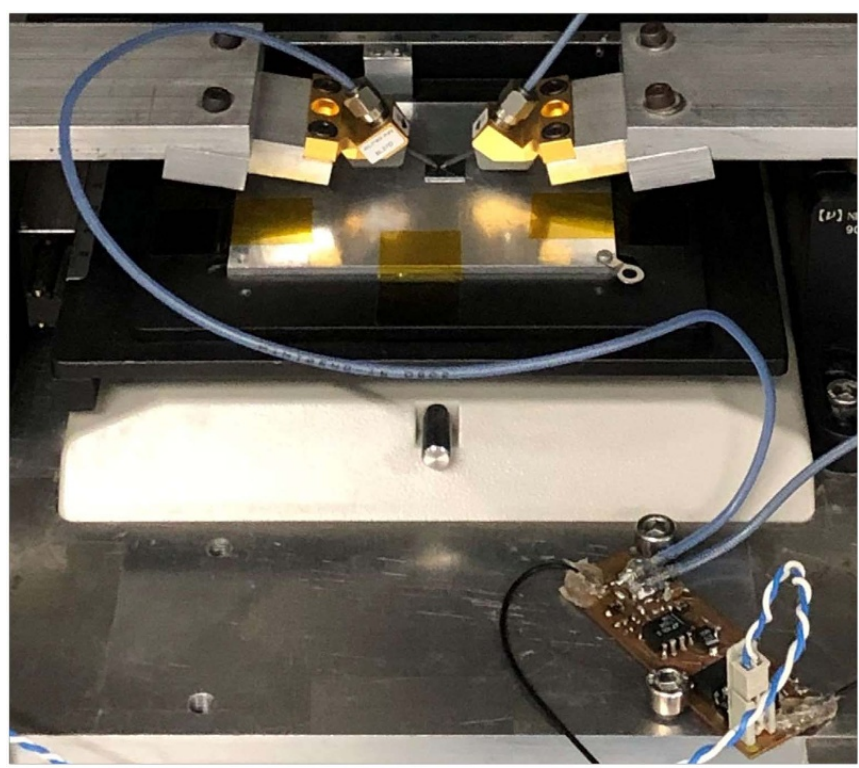

Figure 8. Photo of a coupled-resonator unit connected to a test board through a probe station.

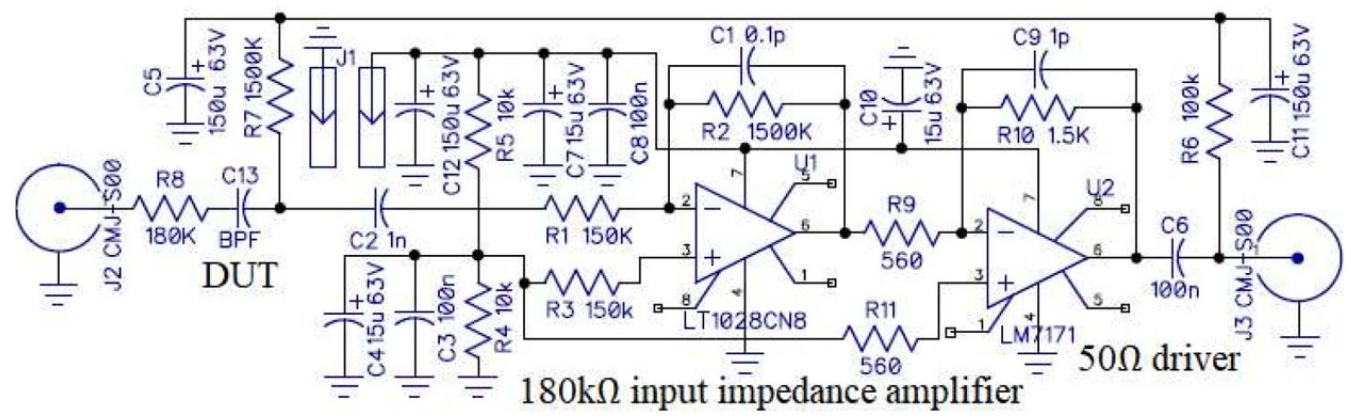

Figure 9. Schematic diagram of the test board.

layer deposition of the PolyMUMPs process, i.e. every layer follows the shape underneath. Therefore, the moving plate layer is not flat as opposed to the model assumption. The circular ridges shown in figure 7 have an effect on the elastic behavior of the moving plate. This effect is not modeled in the equivalent circuit. Figure 7(b) shows the overlap region of the resonators and the shape of the Poly2 layer on top of the gap. The vertical interface at the intersection of the top and the bottom electrodes produces a radial in-plane electrostatic force which is not covered in the model. Also, the sacrificial layer release holes are not present in the equivalent circuit.

\section{Experimental verification}

\subsection{Test setup}

Fabricated coupled-resonator devices are first subjected to DC bias tests utilizing the probe station before the network analyzer measurements under vacuum. We apply a DC bias at the input and output electrodes through a series $1 \mathrm{M} \Omega$ resistor up to $90 \%$ of the expected collapse voltage. Since there is no isolation layer between the Poly 2 and Poly1 layers, collapsing the plate causes electrical short and should be avoided. This test verifies the structural integrity of a device and eliminates the defective devices from further testing.

Following the DC bias tests, we connected the device to a test board via a probe station, as shown in figure 8 . The test board includes a series resistor at the input to provide matched source impedance and a two- stage transimpedance amplifier at the output to drive a $50 \Omega$. The schematic of the test board is given in figure 9. The overall test unit is placed inside a NANOVAK $^{5}$ NVSS-600 vacuum chamber, which has $10^{-7}$ Torr pressure level and several electrical feedthroughs.

\subsection{Measurement results}

S-parameters of the test unit under vacuum are measured using a $50 \Omega$ network analyzer. The measured average dimensions of the sample device under test (DUT) are given in

${ }^{5}$ NANOVAK, Inc., Ankara, Turkey, www.nanovak.com.tr. 
Table 2. Measured dimensions of the DUT.

\begin{tabular}{ll}
\hline Resonator radius, $a$ & $98 \mu \mathrm{m}$ \\
Electrode radius, $a_{0}$ & $80 \mu \mathrm{m}$ \\
Plate thickness, $t_{\mathrm{m}}$ & $1.6 \mu \mathrm{m}$ \\
Gap height, $t_{\mathrm{g}}$ & $700 \mathrm{~nm}$ \\
Overlap ratio, $\alpha$ & 0.043 \\
\hline
\end{tabular}

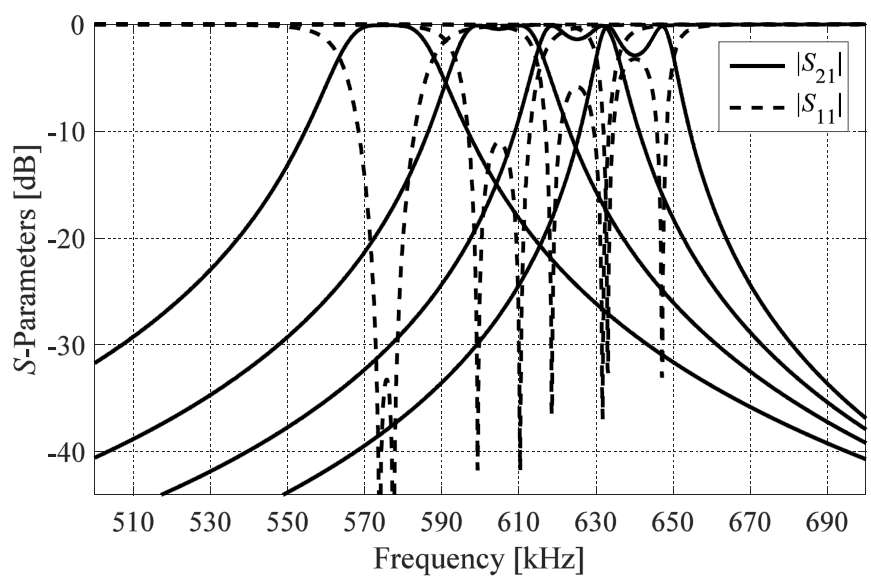

Figure 10. $\left|S_{21}\right|$ (solid line) and $\left|S_{11}\right|$ (dashed line) simulation results of the sample coupled-resonator unit for $V_{\mathrm{DC}}=16 \mathrm{~V}$ up to $22 \mathrm{~V}$ in $2 \mathrm{~V}$ steps (from right to left) under vacuum.

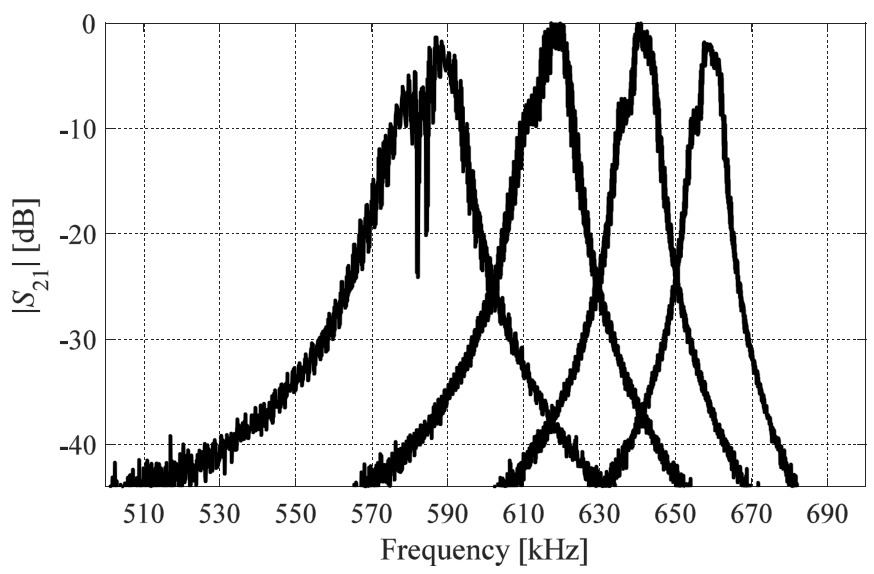

Figure 11. Measurement results of $\left|S_{21}\right|$ transmission characteristics of the sample coupled-resonator unit for $V_{\mathrm{DC}}=16 \mathrm{~V}$ up to $22 \mathrm{~V}$ in $2 \mathrm{~V}$ steps (from right to left) under vacuum.

table 2. S-parameter simulation results under DC bias voltages between $16 \mathrm{~V}$ and $22 \mathrm{~V}$ in $2 \mathrm{~V}$ steps are plotted in figure 10 . There is a frequency shift to lower frequencies under increasing $V_{\mathrm{DC}}$, as expected. Corresponding measurement results of the sample DUT are given in figure 11. We carried out the measurements under a custom-made probe station that fits in the vacuum chamber. This approach brings the coaxial cable connection requirement between the probe station and the test board. The capacitance of this coaxial cable connection deteriorates the SNR of the measurements. In addition, the gap height variations between the resonators cause asymmetry in the transmission response. We observed a device characteristic that complies with the simulation results considering the tolerances of the material properties and the structural differences between the model assumptions and the PolyMUMPs process. We have verified that the model outcome matches the FEM simulations under various dimensional parameters and DC bias conditions. A dedicated process where a flat vibrating plate is produced is instrumental for improved precision in performance predictions.

\section{Conclusions}

We proposed and experimentally validated modeling and design approaches for a mechanically coupled clamped circular plate resonator structure without a separate mechanical coupling element. Low mechanical complexity and high reliability were the main motivations behind the choice of this structure.

The usual practice presented in the literature is to analyze fabricated coupled MEMS resonators using FEM and tune the model parameters accordingly for a specific set of dimensions, bias voltage, and material parameters. This approach makes designing a coupled-resonator filter based on electrical specifications very difficult. We developed a physics-based lumped element equivalent circuit model, which provides insight, accurate and fast simulation results. The model revealed the practical limitations on the center frequency, bandwidth, and termination impedance. We also derived a formula that relates the ratio of the termination impedance to the center frequency for polysilicon material under the specified conditions. Using the provided design procedure, one can determine whether the required filter specifications are satisfied with this type of coupled-resonator structure.

PolyMUMPs process was used for the experimental verification of the model. An optimum design could not be implemented due to the process limitations, such as the gap height, plate thickness, and sacrificial layer release holes. Although the process enforced some structural differences from the proposed model, the measurement results proved the validity of the developed model. Dedicated production processes in which uniform vibration plate can be implemented are useful to have better performing resonators.

As future work, higher-order bandpass filters based on inline or cyclic connections [23, 39] of more than two clamped circular plate resonators can be designed using the given model approach. Cross-coupling between the non-neighboring resonators can also be implemented by placing additional mechanical paths made of a series of in-line coupled resonators.

\section{Data availability statement}

The data that support the findings of this study are available upon reasonable request from the authors.

\section{ORCID iD}

Muhammed Abdulcelil Acar (D) https://orcid.org/0000-00026858-2794 


\section{References}

[1] Mason W P 1939 Electrical and electromechanical system employing magnetostrictive devices 2170206A

[2] Doelz M L 1952 Electromechanical filter 2615981A

[3] George R W 1956 Electromechanical filters for $100 \mathrm{kc}$ carrier and sideband selection Proc. IRE 44 14-18

[4] Hathaway J C and Babcock D F 1957 Survey of mechanical filters and their applications Proc. IRE 45 5-16

[5] Fowler P H 1965 Piezoelectric filter 3189851A

[6] Johnson R A, Borner M and Konno M 1971 Mechanical filters-a review of progress IEEE Trans. Sonics Ultrason. 18 155-70

[7] Johnson R A 1983 Mechanical Filters in Electronics (New York: Wiley)

[8] Linder C, Paratte L, Gretillat M-A, Jaecklin V P and De Rooij N F 1992 Surface micromachining J. Micromech. Microeng. 2 122-32

[9] Howe R T, Boser B E and Pisano A P 1996 Polysilicon integrated microsystems: technologies and applications Sens. Actuators A 56 167-77

[10] Bustillo J M, Howe R T and Muller R S 1998 Surface micromachining for microelectro-mechanical systems Proc. IEEE 86 1552-74

[11] Ayazi F and Najafi K 2000 High aspect-ratio polysilicon micromachining technology Sens. Actuators A 87 46-51

[12] Lin L, Howe R T and Pisano A P 1998 Microelectromechanical filters for signal processing J. Microelectromech. Syst. 7 286-94

[13] Wang K and Nguyen -C T-C 1999 High-order medium frequency micromechanical electronic filters J. Microelectromech. Syst. 8 534-56

[14] Jing Q, Luo H, Mukherjee T, Carley L R and Fedder G K 2000 CMOS micromechanical bandpass filter design using a hierarchical MEMS circuit library Proc. 13th IEEE Int. Conf. Micro Electro Mech. Syst. (Miyazaki, Japan, 23-27 Jan. 2000) pp 187-92

[15] Bannon F D, Clark J R and Nguyen C T-C 2000 High-Q HF microelectromechanical filters IEEE J. Solid-State Circuits 35 512-26

[16] Motiee M, Mansour R R and Khajepour A 2006 Novel MEMS filters for on-chip transceiver architecture, modeling and experiments J. Micromech. Microeng. 16 407-18

[17] Pourkamali S and Ayazi F 2005 Electrically coupled MEMS bandpass filters: part I: with coupling element Sens. Actuators A 122 307-16

[18] Pourkamali S and Ayazi F 2005 Electrically coupled MEMS bandpass filters: part II. without coupling element Sens. Actuators A 122 317-25

[19] Galayko D, Kaiser A, Legrand B, Buchaillot L, Combi C and Collard D 2006 Coupled-resonator micromechanical filters with voltage tuneable bandpass characteristic in thickfilm polysilicon technology Sens. Actuators A $126227-40$

[20] Li -S-S, Lin Y-W, Ren Z and Nguyen C T-C 2006 Disk-array design for suppression of unwanted modes in micromechanical composite-array filters Proc. 19th IEEE Int. Conf. MEMS, Tech. Dig. (Istanbul, Turkey, 22-26 Jan. 2006) pp 866-9

[21] Li -S-S, Lin Y-W, Ren Z and Nguyen C T-C 2007 A micromechanical parallel-class disk-array filter Proc. IEEE Int. Freq. Control Symp. Joint 21 st Eur. Freq. Time Forum (Geneva, Switzerland, 29 May-1 June 2007) pp 1356-61
[22] Shalaby M M, Abdelmoneum M A and Saitou K 2009 Design of spring coupling for high-Q high-frequency MEMS filters for wireless applications IEEE Trans. Ind. Electron. 56 1022-30

[23] Greywall D and Busch P 2002 Coupled micromechanical drumhead resonators with practical application as electromechanical bandpass filters J. Micromech. Microeng. 12 925-38

[24] Köymen H, Atalar A, Aydogdu E, Kocabas C, Oguz K, Olcum S, Ozgurluk A and Unlugedik A 2012 An improved lumped element nonlinear circuit model for a circular CMUT cell IEEE Trans. Ultrason. Ferroelectr. Freq. Control 59 1791-9

[25] Wang K, Bannon III F D, Clark J R and Nguyen C T-C 1997 Q-enhancement of micromechanical filters via low-velocity spring coupling Proc. IEEE Int. Ultrasonics Symp. (Toronto, ON, Canada, 5-8 October 1997) vol 1 pp 323-7

[26] Greenspan M 1979 Piston radiator: some extensions of the theory J. Acoust. Soc. Am. 65 608-21

[27] Timoshenko S and Woinowsky-Krieger S 1959 Theory of Plates and Shells 2nd edn (New York: McGraw-Hill)

[28] Mason W P 1942 Electromechanical Transducers and Wave Filters (New York: D. Van Nostrand)

[29] Leissa A W 1969 Vibration of plates (NASA SP-160, US Government Printing Office)

[30] Kelly P 2013 Solid Mechanics Part II: Engineering Solid Mechanics - small strain 6.8 Plate Vibrations (available at: $\mathrm{http} / / /$ homepages.engineering.auckland.ac.nz/pke1015/ SolidMechanicsBooks/Part_II/06_PlateTheory/ 06_PlateTheory_08_Vibrations.pdf (Accessed 20 Aug. 2021))

[31] Bircumshaw B, Liu G, Takeuchi H, King T J, Howe R, O'Reilly O and Pisano A 2003 The radial bulk annular resonator: towards a $50 \Omega$ RF MEMS filter Proc. 12 th Int. Conf. Solid-State Sens., Actuators, Microsyst. (Boston, MA, USA, 8-12 June 2003) vol 1 pp 875-8

[32] Demirci M U and Nguyen -C T-C 2006 Mechanically corner-coupled square microresonator array for reduced series motional resistance J. Microelectromech. Syst. 15 1419-36

[33] Clark J R, Pai M, Wissman B, He G and Hsu W-T 2006 Parallel-coupled square-resonator micromechanical filter arrays Proc. IEEE Int. Freq. Control Symp. Expos. (Miami, FL, USA, 4-7 June 2006) pp 485-90

[34] Nguyen -C T-C 2007 MEMS technology for timing and frequency control IEEE Trans. Ultrason. Ferroelectr. Freq. Control 54 251-70

[35] Pourkamali S, Ho G K and Ayazi F 2007 Low-impedance VHF and UHF capacitive silicon bulk acoustic wave resonators-part I: concept and fabrication IEEE Trans. Electron Devices 54 2017-23

[36] Forouzanfar S, Mansour R and Abdel-Rahman E 2012 Lorentz force transduction for RF micromechanical filters J. Micromech. Microeng. 22035018

[37] Ziaei-Moayyed M and Howe R T 2010 Higher-order dielectrically transduced bulk-mode ring resonator with low motional resistance Proc. IEEE Int. Freq. Cont. Symp. (Newport Beach, CA, USA, 1-4 June 2010) pp 19-24

[38] Hung L-W and Nguyen C T-C 2012 High-Q Low-Impedance MEMS Resonators UCB/EECS-2012-218 (EECS Department, University of California, Berkeley)

[39] Chivukula V B and Rhoads J F 2010 Microelectromechanical bandpass filters based on cyclic coupling architectures J. Sound Vib. 329 4313-32 